\title{
Keeping Religion in Mind
}

Cognition is the set of processes by which we come to know the world. Cognitive science is the set of disciplines which investigate these processes and propose explanatory theories about them. What cognitive science is discovering is that the relationships of these processes to each other are quite complex and the route to their products intricate. The resulting picture of cognition that emerges from the study of cognitive processes belies any simplistic views of the mind either as a blank slate needing only external input to establish knowledge, or as a general, all-purpose learning mechanism that acquires knowledge in simple, lockstep stages. Instead cognition is thought to involve various predispositions or biases to acquire knowledge in complex ways, and to involve intricate relationships between cultural input and the mechanisms which process it.

For some students of religion it is becoming increasingly clear that cognitive science promises to contribute to an explanatory understanding of aspects of religious ideas and the practices such ideas inform. In fact some cognitive scientists seem to have discovered in the course of their inquiries into the set of processes by which we come to know the world that religion is worth keeping in mind (Boyer 1994; Barrett and Keil 1996; Lawson and McCauley 1990; Lawson and McCauley 1993; Guthrie 1993; Malley 1996; Whitehouse 1992; Whitehouse 1995).

The phrase, "keeping religion in mind", is obviously ambiguous. It can be taken to mean: "Do not forget to study religion as you study the mind." This sense of the phrase is addressed to the inquirer, the one doing the study, and involves the recommendation that the scientist not forget to include religion in the course of her investigation. The phrase can also be taken to mean: "Do not forget to keep religion in the mind rather than in the world." The implication here is that too much attention is being paid to the socio-cultural aspects of religion at the expense of a serious consideration of its cognitive aspects. This mood is particularly evident in the headlong, postmodernist drive to see everything as socio-cultural with the mind considered little more than a kind of yo-yo toy acting only in response to 
socio-cultural forces. The admonishment contained in the recommendation to keep religion in mind reminds us that there is a science of mind, that religious people have minds, use them, and that such minds are worth studying in a systematic and empirical manner. The phrase could also mean that the study of religion should continue to focus on the mind rather than being relegated to the emotions. I intend all three of these interpretations: As you study the mind, do not forget to study religion. Do not be so overwhelmed by socio-cultural factors that you forget about the key role that the mind plays in the formation of religious ideas and the practices they inform. And when you study the formation of religious ideas do not become too easily sidetracked into considering only emotive processes.

Now, just because I recommend keeping religion in mind does not mean that the study of religion should stop there, nor that religion is nothing more than mental machinations. I have little doubt that throughout human history, and across human cultures that the term "religion" has a referent i.e. that there are sets of socio-cultural practices, types of socio-cultural institutions, and forms of sociocultural behavior, that can be called religion and about which there is a great deal to be said, some of it of great interest to scholars interested in developing an explanatory understanding of religion. The danger lies in conceiving of culture as a completely integrated system. As every scholar of religion knows, the moment you start trying to individuate such a wide range of ideas, values, practices, institutions etc., you run into trouble because it is very difficult to know where one set of phenomena ends and another begins. When we talk about "religion" we are actually talking about many types of systems rather than just one, systems which have different properties and are transmitted in different ways. In the light of such complexity it does us well to remember that the reification of religion is an ever present danger. It is also worth noting that linguists began to make theoretical progress when they recognized that the same was true of languages. From a linguistic point of view it is difficult if not impossible to individuate a language. In fact at a certain moment in the theoretical development of linguistics it became pointless to do so. Linguists recognized that the proper subject matter of their disciple was grammar rather than "language". Or more precisely grammar became their theoretical object. In order to clarify this distinction, some linguists have argued for a distinction between external and internal language. External language is a sociocultural entity without clear boundaries. Internal language is a very complicated set of internal mechanisms that process linguistic in- 
formation. And developmental psychologists have learned that the acquisition of knowledge is a complex process in which different concepts are acquired at different phases of cognitive development.

Most scholars of religion are aware of the problem of the difficulty of individuating religion as well as the danger of reifying it. As a consequence they have employed various strategies in order to overcome the problems and the dangers. Some scholars of religion have been driven to search for the core ideas that define religion in specific historical contexts. Their motto seems to be: If you cannot find the whole, then at least look for the center. Others have asserted that religions contain no common features at all. For them, every religion is completely different from every other religion. In fact this approach has led some scholars not to the study religion at all but to the study of uniqueness instead; which is quite a unique approach! Others regard almost anything as a religion. The motto here seems to be if it moves it's religion, if it doesn't move it's religion. There are many obvious advantages to such an approach. You cannot miss your target. Like the perfect hunter, you hit everything that you are aiming at because you never know how the targets differ from each other.

Seductive as these various approaches are, I shall follow none of them for the simple reason that they all confuse the level of analysis. Things can differ with each other at one level and at other levels be remarkably similar. The basis for comparative work lies in the ability to distinguish levels of analysis. For example, languages differ from each other in remarkable ways. Nevertheless they all can be analyzed, for example, in terms of the relationships among the subject, verb and object forms.

It is my view that if we pay attention to what cognitive science is discovering about the mind there is the chance that we will discover how what is in the mind has an effect on what is in the world. However, I would like to keep religion in mind long enough to ensure that we acquire enough knowledge about the cognitive aspects of religion in order to examine their effects on the world, specifically the world of religious practice.

How then shall we proceed? What do we take as our object of study? It is worth rehearsing briefly some of the alternatives. Intellectualists have taken as their object of study the human interest in explaining things. Hence, myths have been of particular concern to them. They have argued that religious ideas about origins and structures are our earliest, if outmoded, and certainly idiomatically different, explanations. Such views have not been confined to scholars of religion but have also found a prominent place in the history 
and philosophy of science as well as anthropology. For example, the anthropologist Robin Horton (Horton and Finnegan 1973) has proposed that the underlying structure of physics is strongly analogous, if not homologous, to the structure of religious mythology. Both count as putative explanations of the world by appealing to hidden variables.

Symbolists have taken as their object of study the human tendency to symbolize what is most important to them in their society. So religious ideas are supposed to reflect, if not mirror, social form. Ideologists have taken as their object of study human power relationships and attempted to show how religious ideas and practices either reinforce or subvert such relationships. Emotivists have taken as their object of study human feeling and attempted to identify religious experience as either a heightened form of feeling, or a radically new form of feeling. And structuralists have taken as their object of study specific types of conceptual relationships, especially those involving patterns of similarity, homology, and opposition and then have attempted to show that religious narratives and ceremonial practices exemplify these properties.

From my point of view all of these theories have made a contribution to an explanatory understanding of religion. I think that we can learn from all of them. They teach us that in religion, explanation, symbolism, power relationships, the affective life, and analogy all play a significant role in social interaction and personal curiosity. Inquiry, however, does not stop with such ideas although it is enlightened by them.

Cognitivist theorists of religious ideas and practices take as their object of study religious representations. Of particular interest is the problem of how religious representations are related to other types of representations. Part of the reason for bringing the mind more clearly into the picture is not that the above-mentioned theories have ignored the mind altogether. Rather, the theory of the mind that intellectualists, symbolists, emotivists, ideologists and structuralists either employ or presuppose is just too simple. Even those theories which do not start with the mind as a blank slate still presume that the processes by which we come to know the world are quite uncomplicated. This is true even for those scholars who talk about the social construction of reality. Seldom do you see much discussion of what kinds of minds are doing in the process of social construction. The view seems to be that all we need do is identify the socio-cultural forces that are at work. Acquisition of knowledge is finally a matter of instruction or socialization: Teach a child to speak, as the child learns to speak, teach her about the world. 
Sooner or later she will know more than she knew at the beginning of the process. So, knowledge involves primarily socio-cultural variables. Minds have to be simple enough to be able to learn and just complex enough to learn different things. Surely more needs to be said than this.

Cognitive science claims to know what that more involves. For example, a four-month-old infant, not yet being able to speak except babble in a rather charming sort of way, can already, without being instructed in the rules of arithmetic and the physical ways of the world, make numerical and physical judgments. In plain language, cognitive and developmental psychologists have good reasons (experimental evidence) for thinking that infants can do simple arithmetic and naive physics before they can talk. What has encouraged cognitive scientists to come to such a preposterous conclusion? Psychologists studying cognitive development have come to these conclusions by designing clever experiments which employ two techniques, dishabituation and preferential looking.

Dishabituation: When an infant is presented with an object to look at she will at first pay attention to it but will eventually look away. When the object is taken away and then shown to her again she will look at it again but for less time, and so on until she will finally almost completely ignore it. The infant has become habituated to the particular object in question. Now show the infant a new object, very similar to the first, but with some significant difference, and she will look at it for a longer time again. The infant has become dishabituated from the first object and her attention captured by the new object. The psychologist, therefore, can measure the interest of the infant by noting the amount of time that the infant spends looking at an object. The amount of time spent looking at an object gives psychologists an important means of measuring the infant's response to various stimuli. In other words we have a way of measuring a four month old's ability to note differences among successively presented objects by gauging the amount of time the infant spends looking at such objects.

Preferential looking. Infants will not only look longer at new objects than at objects they have become habituated to, they will also demonstrate a preference for looking at certain kinds of objects in which physical and numerical principles are apparently violated. Psychologists have no qualms about attributing to infants the notion of surprise by measuring their reactions to the violation of physical and numerical principles because infants will prefer to look at events which do so. Such events go contrary to their expectations and, therefore, rivet their attention. 
We shall take the putative ability to perform certain arithmetical operations as a case in point. Karen Wynn (1992) has performed the following experiment. Infants, five months old, are first shown a hand placing a doll on a stage. Then a screen rises up in the front of the stage and hides the doll. The hand appears from the wings carrying a second doll, places the second doll behind the screen, and leaves it empty so that the infant can see that the doll is no longer in the hand. The screen is then dropped and the dolls on the stage exposed. In one experiment when the screen is dropped there are two dolls on the stage. In another experiment there is only one doll! Wynn discovered that infants preferred to look longer when there was only one doll present than when there should have been two. Her hypothesis is that the infant knows that an arithmetical principle has been violated: $1+1=2$, and not 1 . Wynn has performed similar experiments by changing the number of dolls. If the experimenter, starting with one doll already on the stage, puts two additional dolls down, but when the curtain is raised there are only two dolls on the stage, the infant will look longer than if there were three. $1+2=3$ is not surprising. $1+2=2$ is, both to the infant and to us! Need I remind you that even adults are intrigued by magicians. If I were to start rising to the ceiling right now I am sure that this event would capture your interest.

Other experiments have tested infants for their knowledge of the principles of physical continuity of motion. Infants seem to expect that solid objects move in continuous paths and are surprised when they don't. They also seem to have a principle of solidity. They do not expect one solid object to pass through another. And they also seem to possess a principle of cohesiveness.

Infants also seem to know the difference between mechanical, teleological and cognitive agency (Leslie 1994). Infants will look longer at a ball hitting another ball causing the second ball to roll than at either a stationary ball or a rolling ball. And they will look longer at a ball that starts to roll on its own than at a ball that rolls because it came into contact with another ball. On the basis of such experiments psychologists conclude that infants within the first few months of life know the difference between something that can initiate action willingly and something that can act only when something else causes it to act.

But why mention such experiments which, while interesting, seem to be a long way from the study of religion? I mention them because I think that the concept of agency is crucial for an explanatory understanding of religion. In religion after religion agents with special qualities create the world and destroy it, transcend the world and 
are immanent in it. In fact in religious worlds the number of agents acting on the stage are sometimes mind-boggling in their complexity, number and qualities.

Some scholars have argued convincingly that what distinguishes religious ideas from others is their counter-intuitiveness. They violate our common expectations of what the world is like. This is a good place to start but it is not enough. While many of the properties of religious agents are counter-intuitive the idea of agency is not itself counter-intuitive (except, perhaps, to some philosophers who imagine the world devoid of agency). The idea of agency is fundamental to the traffic that humans have with the world. It should not be surprising therefore that the concept of agency would play a particularly prominent role in religious systems. Perhaps it is only the theologians in various religious traditions who attempt to get beyond the notion of agency to some more esoteric and specialized concept. But that is a very small group of thinkers who by no means are representative of the thinking of religious participants in general. Please note, in any case, that the question is not whether there really are agents in the world, or even whether there are superhuman agents. The question is rather whether all humans have certain basic expectations about the world and whether such expectations include the notion of agency.

It is my view that the concept of agency is particularly important in cognitive studies hence my interest in the early appearance of the concept of agency in infants. My argument is really quite straightforward. If the concept of agency occurs as early in cognitive development as experimental psychology asserts that it is, then it should not surprise us that it will not only be employed in human traffic with the world but adumbrated in special contexts such as ritual action. While we should be surprised that the concept of agency occurs early in cognitive development (and science is supposed to surprise us and, therefore, grab our attention) we should be surprised at the inferences that such a discovery makes possible. We should not be surprised, for example, that religious ideas involving agents with special qualities were to follow naturally from our ordinary ideas of agents.

How then are agents represented? Agents initiate action. They get things done by doing things to other agents and to other objects. Given the notion of an agent that can do things to other agents and objects, it requires very little additional cognitive processing to postulate agents with special qualities. As I have already said, the world of religious ideas throughout history and across cultures is populated with agents with a host of special qualities. Religiously 
conceived agents can be invisible, immortal, know things that no one else knows, move faster than the speed of light, transform things from one type of being into another, rise from the dead. In fact, as Boyer (1994) has argued, these special qualities are so attention-grabbing, and therefore, so easily transmittable, that we tend to overlook those aspects of religious ideas that are quite ordinary. Most of our inferences about them assume perfectly ordinary ontologies.

Thus far we have only attempted to establish the presence and importance of the idea of agency. Now we turn to issues concerning practice. Agents play a particularly important role in religious ritual, religious practice par excellence. In some religious traditions, for example, you cannot regard yourself as married or be treated by others as being married, unless an agent with special qualities has performed a ritual on you and your future spouse. And that agent, in turn, cannot perform the ritual of marriage unless another agent with special qualities has performed a ritual on him or her. The buck only stops with the gods initiating the first ritual, and these initial agents have very special qualities indeed. In fact we have argued elsewhere (Lawson and McCauley 1990) that in order to get a ritual tradition going it is necessary to have agents with special qualities, i.e. Superhuman agents. These agents are variously represented either as the initiators or as the patients of the particular action in question. Their very presence in the religious system makes a difference even when they are described as patients of the action. In rituals such as weddings, in some religions, the immediate agent initiating the action is a ritual official, but unless the imbedded rituals contain a culturally postulated superhuman agent with special qualities who grounds the action, the action will be judged invalid. So it is necessary that a superhuman agent be implicated in the action somehow. It is equally important to find out where in the structural description of the ritual the agent is represented. For example, in a sacrificial ritual the superhuman agent is the patient of the ritual act, the superhuman agent is the recipient and not the initiator of the sacrifice.

The surprising point is that such rituals will require repetition. But where the superhuman agent is the ultimate initiator of the action you will find a ritual that does not require repetition. It is important to note that there is nothing on the surface that gives us any obvious clues about which rituals require repetition and which do not. And it is dangerous to generalize by examining the content of a religious system in order to discover whether it requires repetition or not. In one religious system, for example, baptism might be 
an unrepeated ritual in another it may be repeated many times. We need to examine the formal relationships of the specific ritual in question in order to discover its repeatability. And what is most significant will be where in the structural description the superhuman agent is represented. Similar conclusions can be arrived at by paying attention to whether or not a ritual can be reversed, will permit substitution, is effective, well-formed or relatively central to the religious system. What is important here is that the kinds of judgments religious ritual participants will make about the form of their rituals shows that we are dealing with a system of judgments guided by certain principles. Knowing what these principles are permits us to make various predictions. For example, a religious system that abandons or loses its unrepeated rituals will either have to create new ones or fade away. We have contemporary evidence from ethnographic studies especially the work of Frederick Barth $(1975,1987)$ and Harvey Whitehouse $(1992,1995)$ that supports this prediction.

A cognitive approach to the study of religious ritual demonstrates that when you examine religious ideas and the practices they inform you are looking at a religious system in operation. The relationships among such ideas are systematic and orderly. If they were not we would be looking at a random array of ideas and practices. In such a situation anything would go. But in religious systems anything does not go. The judgments that religious ritual participants make about their own systems are informed by underlying principles that are part of their implicit knowledge. Perhaps, most significantly, such implicit knowledge does not seem to be acquired by instruction. So rather than looking primarily at social and cultural facts in order to explain their acquisition we also need to start looking more closely at how the human mind works; we need to be developing a new psychology of religion as a subdiscipline of cognitive science.

It should now be apparent why I said earlier that it is important to acknowledge the discovery of cognitive science that the human mind is not a general, all purpose learning mechanism which only needs information via instruction to get the cognitive juices flowing. In fact it is becoming increasingly clear that cognition consists of a set of specialized processes with different kinds of products. These mechanisms do not come on line all at once. They require special information for their activation. In saying that I must insist that there is no need to think that religious ideas themselves are part of the early or even initial conditions of the human mind. All we need to acknowledge is that the ordinary cognitive resources that we bring into the world, or at least develop very early, are capable of 
being employed for bringing about and apprehending religious ideas and the practices they inform. Seeing that most of us are born into a world in which such religious ideas already have currency, and such religious practices are a relatively frequent occurrence, it is no surprise that the set of cognitive processes by which we come to know the world stand ready and willing to be employed in the service of conceiving agents with special qualities and the practices that such conceptions inform. What is surprising is the principles involved, and the hidden relationships revealed. It is worth keeping religion in mind in order to discover the principles and the relationships they disclose.

\section{References}

Barrett, Justin, and F. Keil.

1996 Conceptualizing a Non-Natural Entity: Anthropomorphism in God Concepts. Cognitive Psychology 31: 219-247.

\section{Barth, Fredrik}

1975 Ritual and Knowledge among the Baktaman of New Gunea. New Haven: Yale university Press.

1987 Cosmologies in the Making: A Generative Approach to Cultural Variation in Inner New Guinea. Cambridge: Cambridge University Press. (Cambridge Studies in Social Anthropology, 64)

Boyer, Pascal

1994 The Naturalness of Religious Ideas: A Cognitive Theory of Religion. Berkeley: University of California Press.

Guthrie, Stewart Elliott

1993 Faces in the Clouds: A New Theory of Religion. New York: Oxford University Press.

Horton, Robin, and Ruth Finnegan (eds.)

1973 Modes of Thought: Essays on Thinking in Western and Non-western Societies. London: Faber \& Faber.

Lawson, E. Thomas, and Robert N. McCauley.

1990 Rethinking Religion: Connecting Cognition and Culture. Cambridge: Cambridge University Press.

1993 Crisis of Conscience, Riddle of Identity: Making Space for a Cognitive Approach to Religious Phenomena. Journal of the American Academy of Religion 61: 201-223.

Leslie, Alan

1994 A Theory of Agency. In: Dan Sperber, David Prem,ack and Ann James Premack (eds.), Causal Cognition: A Multidisciplinary Debate. New York: Oxford University Press. 


\section{Malley, Brian}

1996 The Emerging Cognitive Psychology of Religion: A Review Article. Method \& Theory in the Study of Religion. 8: 109-141.

Whitehouse, Harvey

1992 Memorable Religions: Transmission, Codification and Change in divergent Melanesian Contexts. MAN 27: 777-797.

1995 Inside the Cult: Religious Innovation and Transmission in Papua New Guinea. Oxford: Clarendon.

Wynn, Karen

1992 Evidence against Empiricist Accounts of the Origins of Numerical Knowledge. Mind \& Language 7: 314-332 